\title{
Procrastinación: Revisión Teórica
}

\section{Procrastination: Theoretical Review}

\author{
Candy Atalaya Laureano ${ }^{1}$ \\ Institución Educativa Ing. Carlos Lisson Beingolea \\ Lupe García Ampudia ${ }^{2}$ \\ Universidad Nacional Mayor de San Marcos
}

Recibido: $04-10-19$

Aceptado: $17-12-19$

\section{Resumen}

El objetivo del artículo es analizar las perspectivas actuales sobre el concepto de procrastinación, los tipos y modelos teóricos que respaldan a la variable en mencionada. Se revisó metódicamente las fuentes bibliográficas sobre la variable; asimismo se eligieron artículos, reportes de investigación y estudios psicométricos de diferentes revistas científicas de psicología y ciencias sociales indexadas en bases de datos electrónicas a nivel nacional e internacional. Los resultados muestran múltiples acepciones para definir con exactitud el significado de la procrastinación, los tipos de procrastinación, sus modelos teóricos y estrategias de prevención en el ámbito educativo. Finalmente, se concluye que a través de una sustanciosa revisión teórica se obtuvo un concepto actual para la comprensión de la variable en estudio.

Palabras clave: Procrastinación; conceptualización; modelos teóricos y estrategias.

\begin{abstract}
The objective of this article is to analyze the current perspectives on the concept of procrastination, its types and its theoretical models which can support the variable we are talking about. Performing a systematic review of the literature about it, likewise, there were selected articles, research reports and psychometric studies published in scientific journals of psychology indexed national and international level at electronic database. The results show multiple meanings to define exactly the meaning of procrastination, its types, its theoretical models and the prevention strategies in the educational field. Finally, it concludes that through a substantial theoretical revision, it was obtain an actual concept to the comprehension of the variable under study.
\end{abstract}

Keywords: Procrastination; conceptualization; theoretical models and strategies.

1 Psicóloga educativa. Institución Educativa Ing. Carlos Lisson Beingolea - La Molina.

E-mail: candyatalayal@gmail.com

2 Asesora de tesis. Directora General de Estudios de Posgrado. Universidad Nacional Mayor de San Marcos. E-mail: lgarciaa@unmsm.edu.pe

(C) Los autores. Este artículo es publicado por la Revista de Investigación en Psicología de la Facultad de Psicología, Universidad Nacional Mayor de San Marcos. Este es un artículo de acceso abierto, distribuido bajo los términos de la licencia Creative Commons Atribucion - No Comercia_Compartir Igual 4.0 Internacional. (http://creativecommons.org/licenses/by-nc-sa/4.0/) que permite el uso no comercial, distribución y reproducción en cualquier medio, siempre que la obra original sea debidamente citada. 
El término procrastinación se ha convertido en un fenómeno típico en nuestra sociedad, cuya actividad de posponer tareas es un acto irresponsable del sujeto. Éste actuar puede ser voluntario o involuntario que genera severas consecuencias, se inicia en la adolescencia y se establece en la edad adulta perjudicando su desempeño académico y laboral (Álvarez, 2010). Antiguamente, se referían a la procrastinación como el comportamiento de postergar tareas, esta conducta era socialmente aceptada y justificada; sin embargo, comenzó a tornarse negativo en la modernidad, cuando la aplicación de técnicas productivas se convirtieron en la fuente de progreso económico y financiero de la sociedad (Steel, 2007).

La procrastinación se desarrolla porque las personas prefieren realizar actividades que les generen resultados satisfactorios a corto plazo en lugar de aquellas que impliquen efectos positivos a largo plazo (Riva, 2006). Asimismo, la procrastinación se ha convertido en un factor trascendente que repercute negativamente en el aspecto académico de los estudiantes; ya que, los adolescentes postergan sus tareas escolares por otras actividades de interés como los juegos, las fiestas, los paseos, entre otros (Contreras-Pulache, Mori-Quispe, Lam-Figueroa, Hinostroza-Camposano, Rojas-Bolivar, Espinoza-Lecca, Torrejon-Reyes y Conspira-Cross, 2011).

En el Perú, se realizó un estudio en Lima Metropolitana cuya evidencia fue que el $20 \%$ de los estudiantes de secundaria practicaban la procrastinación $(n=235)$, sin reportar diferencias por sexo; es decir, los hombres y las mujeres procrastinan con porcentajes similares (Álvarez, 2010). Así también, Valle (2017), investigó sobre los niveles de procrastinación académica en estudiantes de secundaria en una institución educativa de Lima: la muestra estuvo conformada por 130 estudiantes (masculino y femenino) cuyas edades oscilaban entre 12 y 18 años de edad, y los resultados permitieron concluir que existe un nivel alto $(32,3 \%)$ de procrastinación académica en los estudiantes (Valle, 2017). ContrerasPulache, et al, (2011), exploraron el fenómeno de procrastinación académica y la asociación con el apoyo familiar y uso del internet en estudiantes de secundaria en Lima Metropolitana, la muestra estuvo compuesta por 292 estudiantes $(66.1 \%$ varones y $33.9 \%$ mujeres), y los resultados señalan que existe una asociación estadísticamente significativa entre la procrastinación académica, plan de vida, apoyo familiar y uso del internet.

A nivel internacional, se revisaron investigaciones como el análisis de Sirois y Pychyl (2013), investigadores del Reino Unido, quienes concluyeron que la procrastinación puede ser entendida como el predominio de satisfacción del estado de ánimo, pero a corto plazo. Rosario, Costa, Nuñez, Gonzales-Pienda, Solano y Valle (2009), asociaron la procrastinación académica con variables personales, sociales y familiares, la muestra estuvo conformada por estudiantes del séptimo a noveno grado que asisten a los últimos tres años de un instituto en España, se organizó en dos muestras independientes de 580 (47.8\% varones y 
$52.2 \%$ mujeres) y 809 ( $48.6 \%$ varones y $51.4 \%$ mujeres) con un rango de edad entre 11 y 17 años, y los resultados revelaron que la procrastinación disminuye cuando los padres tienen mejor educación; por otro lado, la procrastinación aumenta según los años cursados por los estudiantes, lo cual repercute negativamente sobre su rendimiento escolar.

En suma, la procrastinación no solo se evalúa en el aspecto académico; también influye en la actividades de la vida diaria, debilitando la autonomía del individuo con el incumplimiento de sus obligaciones y deberes domésticos; lo cual, afecta en su desenvolvimiento e interacción al sistema social (Busko, 1998). Asimismo, éste patrón de comportamiento se caracteriza por aplazar voluntariamente la realización de actividades que deben ser entregadas en un momento establecido. Por lo tanto, la procrastinación es un fenómeno que ocupa todas las esferas de acción de la persona; por ello el presente artículo permitirá precisar el concepto, los tipos de procrastinación, los modelos que respaldan al concepto, y variables relacionadas al estudio en mención. Además, en el campo educativo, se aportará información relevante que lleve al análisis y reflexión con el fin de mejorar la calidad de servicio, a través de estrategias y planes de acción que contribuyan al progreso y revaloración del educando.

\section{MÉTODO}

\section{Diseño}

Se revisó metodológicamente diferentes artículos publicados en revistas científicas digitales de Psicología de Dialnet, Redalyc, Psicothema y Scielo indexadas en bases de datos electrónicas como PROQUEST, SCOPUS, ERIC, GALE, SCIENCEDIRECT, SPRINGER, RENATI, ALICIA Y CYBERTESIS.

\section{Instrumento}

Las publicaciones seleccionadas fueron las siguientes: Revista de la Universidad Peruana Unión, Revista Peruana de Epidemiología, Studiositas, Propósitos y Representaciones, Revista Vanguardia de Psicología Clínica: Teórica y Práctica, Revista Iberoamericana de Psicología: Ciencia y Tecnología, Revista Persona, Acción Psicológica, Revista de educación Psicológica, Revista de investigaciones, Temática Psicológica, Revista Gaceta, Journal of Theory and Practice in Education, The Spanish Journal of Psychology, Social and Personality Psychology Compass, Journal of counseling psychology, Journal of Educational Psychology, Psychological Bulletin. También, se revisó tesis de postgrado de la Pontificia Universidad Católica del Perú, Universidad Privada Antenor Orrego, Trujillo., Universidad César Vallejo, Universidad Inca Garcilaso de la Vega, Pontificia Universidad Javeriana de Bogotá, la Universidad Cooperativa de Colombia, Santa 
Marta, Universidad de Valencia y University of Guelph, Ontario, y editoriales como Editorial Paidós, New, Plenum Press, y The New Work Times.

\section{Procedimiento}

Se revisaron artículos desde el año 2009 hasta la actualidad, se utilizaron los siguientes descriptores: procrastinación, procrastinación general y procrastinación académica, en idioma español e inglés. Se incluyeron artículos científicos, reportes de investigación y tesis. Posterior a la lectura y sistematización de la información, se categorizó en cuatro tópicos para profundizar y analizar el tema.

\section{Resultados}

Una revisión al detalle en castellano e inglés, permitió seleccionar 40 textos, dejando los resúmenes y abstracts publicados. Se basó en la variable mencionada en este artículo, y se obtuvo cuatro categorías: (a) desarrollo conceptual de la procrastinación, (b) tipos de procrastinación, (c) modelos teóricos y (d) estrategias de prevención en el ámbito educativo.

\section{DESARROLLO CONCEPTUAL DE LA PROCRASTINACIÓN}

A través de un análisis y reflexión sobre los antecedentes históricos de la humanidad en relación al origen del concepto de procrastinación, se encontró que en el año 3000 antes de Cristo, los egipcios utilizaron el término procrastinación para referirse a la "costumbre de evitar el trabajo y al estado de pereza de una persona ante una actividad necesaria para subsistir" (Steel, 2007, p. 66). Asimismo, en el año 1790 antes de Cristo, el conocido Rey de Babilonia, Hamurabi, consideró a la procrastinación dentro de sus leyes para establecer una fecha límite en la presentación de las quejas, pues reconoció que las demoras eran perniciosas y debían ser contrarrestadas (Steel, 2007). En el año 1400 antes de Cristo, las personas lucharon por la gestión básica del tiempo, Ronald Leprohon, un egiptólogo, tradujo un jeroglífico que dice "amigo, deje de posponer el trabajo y permítanos volver a casa a tiempo" (Steel, 2007, p. 66). Mientras que, para los romanos, la procrastinación era la actividad de espera para contemplar la presencia de los enemigos en los conflictos militares (Álvarez, 2010, p. 161). De igual manera, uno de los antiguos griegos, el poeta Hesiódico escribió, alrededor de los años 800 antes de Cristo, la siguiente declaración sobre la procrastinación "no abandones la labor de hoy para mañana ni pasado mañana, porque un trabajador lento no llena su granero, ni uno que posterga su trabajo" (Steel, 2007, p. 67); es decir, expresaba la relación de la ruina con el aplazamiento del trabajo y las tareas. Cerca de los 400 años después, un ilustre historiador y militar ateniense, Túcicides, señaló que la prórroga solo tenía ventaja para las guerras, brindó importancia a los atributos en la personalidad de los soldados y resaltó que la procrastinación era un rasgo crítico en el carácter. Del mismo modo, un personaje de la historia greco-romana, 
Cicerón, en el año 44 antes de Cristo, formuló que la procrastinación y la lentitud eran de las más despreciables características del ciudadano. En el siglo XVI, la palabra procrastinación tenía una connotación distinta, ya que significaba dejar de hacer las tareas de forma voluntaria sin ser conscientes que esa dilación podría perjudicarles; mientras que, en el siglo XVII, Anthony Walker, un reverendo griego, unió los términos anteriores y aportó en un concepto original sobre la procrastinación, cuya interpretación era aquello que los eruditos y fervorosos consideraban merecedor de repudio y ante lo cual había que luchar (Steel, 2007); es decir, se trataba de una relación íntima entre la evitación de hacer las tareas, la falta de voluntad y el pecado(Álvarez, 2010). No obstante, Kachgal (2001 citado por Angarita, 2012), mencionó que antes de la revolución industrial, el acto de posponer actividades era vista con objetividad, y a nivel social era entendida como una conducta justificada.

Después de identificar los antecedentes antiguos provenientes de distintos contextos socio-culturales, se entiende que el concepto de procrastinación se ha presentado con frecuencia a lo largo de la historia, y era considerada como algo usual en la conducta humana (Ferrari y Díaz-Morales, 2007; Ferrari, Johnson y McCown, 1995). Asimismo, a través de un profundo análisis, la procrastinación comienza a tomar un matiz negativo a partir de la modernidad, cuando los sistemas productivos se convirtieron en la base del desarrollo económico de la sociedad (Steel, 2007). Por lo tanto, el término procrastinación resulta perjudicial cuando el crecimiento de la sociedad depende de la productividad.

La definición etimológica de la procrastinación deriva del verbo en latín procrastināre, que significa dilatar la presentación de una actividad de forma voluntaria, también deriva de la palabra en griego antiguo akrasia, significa hacer algo en contra del juicio o hacerse daño a uno mismo (Steel, 2007, p. 65). Según Steel (2007), la autoconciencia es una pieza fundamental para inferir las consecuencias perjudiciales al sujeto, pues no solo se hace consciente que se está postergando la tarea, sino que hacerlo es una desagradable experiencia y, aun así, las personas lo hacen parte de su vida; entonces, la procrastinación se caracteriza por la falta de regulación de emociones en la gestión de tiempo destinada para una actividad; de esta manera, la procrastinación se interpreta como retrasar algo hasta mañana, y se define como "el hecho de posponer la culminación de una tarea que usualmente resulta en un estado de insatisfacción o malestar subjetivo" (Ferrari et al., 1995). En el verbo inglés "procrastinate", combina el adverbio común "pro" que significa "hacia adelante" con la terminación "cras" o "crastinus" que significa "para mañana", lo cual en conjunto significa aplazar intencional y habitualmente algo que debería ser hecho (Schouwenburg, 2005 citado por Alegre, 2014, p. 63). En ese sentido, la procrastinación puede ser comprendida como el aplazamiento voluntario de los compromisos personales pendientes, a pesar de ser consciente de las consecuencias negativas. Phillip Stamhope (1749 citado por 
Steel, 2007, p. 64) hace mención en su célebre frase "No vagancia, no holgazanería, no procrastinación"; es decir, "nunca dejes para mañana lo que puedes hacer hoy", por su parte Samuel Johnson (1751 citado en Alegre, 2014, p. 63) describió a la procrastinarían como "la locura de retrasar lo que se sabe finalmente que no se puede", siendo esta una de las limitaciones, pues a pesar de un pensamiento moralista y los cuestionamientos de la razón, prevalecen en la mente de las personas; otro estudio, elaborado por Milgram en 1992 (Angarita, 2012), mencionó que las personas se comprometen con actividades a corto plazo, esto ocasiona que procrastinen; asimismo, en las ciudades de desarrollo tecnológico, se evidencia mayor índice de compromisos y fechas límites que hacen que la procrastinación sea aún más elevada (Ferrari y Díaz-Morales, 2007); mientras que, Busko (1998) define la procrastinación como una tendencia a postergar siempre o casi siempre una actividad; o prometer efectuarla más adelante por medio de justificaciones o excusas con el propósito de evitar cierta culpabilidad frente a las tareas.

En la actualidad la Real Academia de la Lengua Española, en la vigésimo segunda edición de su Diccionario de la Lengua Española (2008), define "procrastinar" como "diferir, aplazar"; es decir, las personas con estas conductas muestran intensión de realizar la tarea encomendada, la misma que se posterga para un mañana (García-Ayala, 2016 y Steel, 2011); asimismo, se puede definir la procrastinación como un fenómeno que afecta a las personas directamente en la práctica de sus valores, pues se niegan a realizar una tarea en el tiempo oportuno, dejando de lado una responsabilidad necesaria (Balkis y Duru, 2009), y Ramírez, Tello y Vásquez (citado por Carranza y Ramírez, 2013, p. 96) mencionan que la procrastinación se distingue por la acción de posponer responsabilidades de manera voluntaria, a pesar que en una primera instancia, la intención es de hacerla.

En resumen, la procrastinación es el resultado de carencias en la autorregulación, que conduce al individuo a la dilatación voluntariamente pero inconsciente sobre el inicio y consumación de las tareas o actividades previstas, a pesar de conocer las consecuencias negativas de postergar; asimismo, si en la etapa de la adolescencia estos hábitos están instaurados, es decir, las conductas de procrastinación se mantienen, esto no solamente influye en su desempeño académico, sino, que a largo plazo se verá reflejado en actividades familiares, sociales, laborales entre otras (Quant y Sánchez, 2012; Chan, 2011). Todas estas definiciones planteadas a lo largo de la historia, demuestran que la procrastinación ha sido considerada un modelo inadecuado en el comportamiento humano.

\section{TIPOS DE PROCRASTINACIÓN}

A través de la literatura se puede distinguir dos tipos de concepto relacionados a la procrastinación: General y Académica, que se desarrollará en el siguiente apartado. 


\section{Procrastinación general}

La procrastinación es un constructo de varios significados, ya que presenta múltiples definiciones según el enfoque teórico del estudio. Para Ferrari et al. (1995), la procrastinación general es el hecho de posponer una tarea de poco interés que genera malestar subjetivo; también, este tipo de procrastinación es considerado por la preferencia de establecer relaciones sociales que el enfoque a sus tareas académicas. De esta manera, el procrastinador es aquel que sabe lo que quiere hacer, pero que en realidad no lo hace, y demora en realizar las tareas que deben ser completadas en un periodo de tiempo (Ferrari et al., 1995 y Angarita, 2012). Ferrari y colaboradores (1995) proponen siete tipos referidos a la procrastinación académica: a) perfeccionista, quién aplaza las tareas por incumplimiento de estándares personales; b) soñador, prefiere divagar en fantasías; c) preocupado, temeroso de las consecuencias, pero no se involucra; d) generador de crisis, quien disfruta de la interacción social sin desarrollar las actividades; e) desafiante, responsabiliza a otros de forma impulsiva; f) ocupado, quien atiende varias tareas a la vez y no termina; y g) relajado, aquel que evita situaciones que le generen estrés y compromiso (Ferrari et al.,1995; Natividad, 2014). Asimismo, se ha observado que estudiantes procrastinadores inician sus estudios con retraso por la discordancia de su conducta o hábitos de estudio (Milgram, Sroloff y Rosenbaum, 1988 citado por Steel, 2007, p. 68), en consecuencia, estos sujetos prefieren abarcar su tiempo en actividades sociales que escolares, cuya consecuencia son altos niveles de ansiedad y preocupación, pero no necesariamente trae consecuencias negativas ya que finalmente se llegan a cumplir (Álvarez, 2010).

\section{Procrastinación académica}

Es definida como la tendencia a dejar de lado las actividades hasta una futura fecha, los implicados en presentar problemas de este tipo son los estudiantes (Ellis y Knaus, 2002); cuya consecuencia es un bajo rendimiento académico y deserción escolar (Semb, Glick y Spence, 1979 citado en Steel, 2007, p. 69); además, Rothblum, Solomon y Murakami (1986) mencionan que la procrastinación académica implica un alto nivel de ansiedad debido a la persistente demora en las tareas académicas; mientras que Johnson y Bloom (1995 citado por Balkis y Duru, 2009, p. 20) presentan dos diferentes líneas de investigación para definir a la procrastinación, una de ellas es la responsabilidad en el cumplimiento de tareas y la otra relacionada a las características de su personalidad. Ferrari e investigadores (1995), explican que la procrastinación académica es la demora voluntaria para realizar las responsabilidades académicas, a pesar que la intención de los estudiantes fue de realizar una actividad académica dentro del plazo establecido (Klassen, Krawch, Lynch y Rajani, 2007 citado por Quant y Sánchez, 2012, p. 53). Por otra parte, Quant y Sánchez (2012) manifiestan que en el área educativa "las personas dejan de hacer sus actividades académicas cuando la fecha de entrega está muy cerca, de igual forma suelen realizar muy pocas actividades y evitan 
asumir responsabilidades en el trabajo de grupo" (p. 52), pues el estudiante tiene la idea errónea sobre que la presión impulsa un desarrollo óptimo en sus trabajos. Rothblum, Solomon y Murakami (1986) definen la procrastinación académica como "la tendencia a aplazar siempre o casi siempre una actividad académica, y siempre o casi siempre experimentar ansiedad asociada a la procrastinación" ( $\mathrm{p}$. 390); asimismo, se evidencian dos dimensiones de la procrastinación académica: a) autorregulación académica, que mide el nivel cómo los estudiantes regulan y controlan sus pensamientos, motivaciones y conductas, a través, de un proceso activo y constructivo de respuestas, dirigidas a la consecución de metas en el aprendizaje (Álvarez, 2010, p. 166; Valle, 2017); y b) postergación de actividades, que mide el grado en que los estudiantes retrasan o posponen las tareas académicas, sustituyendo las actividades por otras más agradables que no demanden mucho esfuerzo, y por lo general origina una insatisfacción o malestar subjetivo (Álvarez, 2010, p. 166; Dominguez y Centeno, 2014, p. 13).

\section{MODELOS TEÓRICOS DE LA PROCRASTINACIÓN}

\section{Modelo psicodinámico}

Para Baker, la teoría psicoanalítica y psicodinámica es la más antigua para explicar el comportamiento de manera general (Ferrari, Johnson y McCown, 1995), y hace referencia a que la procrastinación proviene desde la infancia; ya que, en esta etapa se juega un rol muy importante en el desarrollo de la personalidad del adulto y la influencia de los procesos mentales inconscientes y conflictos internos en la conducta. Freud (citado por Ferrari et al, 1995, p. 22) explicó que la acción de postergar está basada en el concepto de evitar tareas, y argumentó que la ansiedad sirve como una señal de advertencia para el ego, de acuerdo con esto las tareas se evitan porque son una amenaza, y estas actividades peligrosas son difíciles de ejecutar. Por otro lado, los teóricos psicoanalíticos coinciden en que las expectativas de los padres influyen en los rasgos de personalidad del individuo y en el desarrollo de la procrastinación; de este modo, los estudiantes pueden sentirse extremadamente presionados por la aprobación de sus padres coaccionada a buen rendimiento académico y a las altas expectativas que tienen sobre ellos; por eso, los adolescentes optan por una postura rebelde y ponen a prueba sus propios límites a través de la procrastinación (citado por Ferrari, et al., 1995, p. 238). Por tanto, los orígenes de la procrastinación son relacionados con asuntos de inmadurez y rebeldía, Sommer (1990 citado por Ferrari y Díaz-Morales, 2007, p. 93) manifestó que la procrastinación tiende a ser un comportamiento de rebelión en relación a la figura paterna o materna (o de autoridad); así que, la procrastinación académica se contempla en términos de transferencia inconsciente en la relación padres e hijos o, en el contexto educativo, la relación profesor-estudiante.

El modelo manifiesta que la procrastinación es el miedo de no cumplir con los objetivos propuesto, por este motivo los sujetos abandonan sus quehaceres a pesar 
de tener habilidades para lograr lo que se proponen. Se puede inferir que el temor de un niño se inicia a partir de las consecuencias desfavorables de sus actos, por lo que terminan postergando sus quehaceres; asimismo, se ha observado que en la adolescencia, esta característica es una constante, cuyo tiempo es desvalorado, lo que genera una sensación de que "los días pasan rápido", esta idea persiste en la adultez joven o juventud, alrededor de los 20 años, donde los malestares son reemplazados por la idea de insuficiencia para realizar o aprovechar las oportunidades, generando altos niveles de ansiedad (Baker, 1979 citado por Steel, 2007, p. 65). Por último, esta teoría fue la primera en relacionar las conductas con la suspensión de los quehaceres.

\section{Modelo Motivacional}

Es un modelo que incita al éxito, visto como indicador inmutable del sujeto que cambia un conjunto de comportamientos para lograr el triunfo en diferentes aspectos de su vida; por ende, el sujeto escoge entre dos enfoques: la fe de lograr el triunfo o al temor a no alcanzar el triunfo; lo primero hace hincapié al factor motivacional y lo segundo a la motivación para evadir un contexto que el sujeto toma como negativo. Cuando prevalece el temor de no cumplir las metas, los sujetos eligen situaciones donde saben que el triunfo está asegurado, dejando de lado aquellas situaciones que son difíciles de alcanzar (Ferrari et al., 1995). Asimismo, McClelland (citado por Rothblum, Solomon y Murakami, 1986, p. 390) destaca que el modelo motivacional analizó la variable procrastinación, que permitió identificar dos teorías: La primera es denominada autodeterminación, que implica un sentimiento de libertad al hacer aquello que uno ha decidido llevar a cabo, y está compuesta por la motivación intrínseca y extrínseca; asimismo, existen evidencias que la motivación autodeterminada tiene una relación negativa con la procrastinación académica; es decir, los escolares que se encuentran desmotivados tienen mayor probabilidad de procrastinar frente a los estudiantes motivados, pues los sujetos al implicarse con una actividad de elección personal es más fácil que la resuelvan, ya que han generado un vínculo de logro. La segunda es la teoría de las metas de logro, que en las últimas décadas ha adquirido mayor importancia en el marco teórico, pues las metas de logro pueden definirse como un comportamiento dinámico orientado a una competencia basada en la activación, cariño, desarrollo de funciones cognitivas y el compromiso de un comportamiento asertivo para ejecutar sus tareas (Elliot, 1999 citado por Wolters, 2003, p. 182). Esta teoría reafirma que la procrastinación dispone de características autolimitantes en las personas (Ferrari y Tice, 2000 citado por Wolters, 2003, p. 184).

En general, este modelo motivacional señala que los estudiantes procrastinadores se encuentran desmotivados, y están propensos a adoptar una postura de insatisfacción en relación a los objetivos que pretenden cumplir o se desaniman cuando obtener un logro implica esfuerzo y dedicación; ante ello, es más probable que opten por suspender o prorrogar el inicio o avance en sus tareas. 


\section{Modelo Conductual}

Skinner (1977 citado por Skinner y Belmont, 1993, p. 160), propone un modelo conductual, el cual manifiesta que una conducta se conserva cuando es repetitiva, y permanece por sus efectos de recompensa; por tanto, desde este modelo, la procrastinación es la práctica que hace el sujeto al ejecutar tareas que impliquen satisfacción a corto plazo; por eso, los estudiantes dejan de realizar sus actividades escolares porque implica un logro a largo plazo, a su vez este comportamiento postergatorio es reforzado implicitamente obtenido "premios" en breve tiempo (Ferrari y Emmons, 1995; citado por Chan, 2011, p. 54); además, este modelo afirma que la procrastinación es dejar las actividades que son poco atractivas para un después (Quant, y Sánchez, 2012).

Es decir, las experiencias previas de los individuos son aprendidas desde la infancia, y se fortalecen a lo largo del tiempo, ya que han sido condicionadas a posponer una actividad por una recompensa a corto plazo, esto conlleva a retrasar los esfuerzos de desarrollar una tarea (Carranza y Ramírez, 2013). A partir de esto, se entiende que los procrastinadores son aquellos sujetos que están acostumbrados a posponer una actividad que requiera de tiempo, el cual genera malestar; por otra actividad que implique un desarrollo rápido con premios inmediatos.

\section{Modelo Cognitivo}

Esta teoría señala que los procrastinadores tienen un procesamiento de información disfuncional, y que constantemente reflexionan sobre su comportamiento de aplazar actividades, por lo que presentan estructuras desadaptativas en su pensamiento, el cual presenta una implicancia negativa de imposibilidad y temor al rechazo social al momento de realizar una tarea (Wolters, 2003). Por otro lado, Wolters (2003), refiere que los sujetos presentan un pensamiento obsesivo cuando no cumplen sus metas propuestas, y manifiestan un pésimo desarrollo de sus actividades, pues tienen dificultad para proyectar y organizar su tiempo, por lo que fracasan constantemente (Stainton, Lay y Flett, 2000 citado por Wolters, 2003, p. 182). Así que, Albert Ellis, en su teoría de la terapia racional emotiva, propuso el modelo $A B C$ para complementar el estudio de la procrastinación. El fundamento principal de este modelo es el contenido y la forma de pensar de la persona; es decir, la manera de interpreta circunstancias y las creencias que ha desarrollado sobre sí mismo, las demás personas y el mundo en general. El modelo $A B C$ establece que $\mathbf{A}$ representa el evento activo, el cual puede ser real o imaginado, $\mathbf{B}$ constituye las interpretaciones o creencias respecto a ese evento, y C las consecuencias (Ellis, 1999); por ejemplo, si ante la petición del profesor de preparar una exposición la persona piensa (A1) "no sirvo para eso", (B1) "me sentiré ansioso y procrastinaré" y (C1) "soy un tonto" o "nunca podré cambiar" (Ellis, 1999). Por otro lado, Ellis y Knaus (2002), postularon a desarrollar la teoría Racional Emotiva Conductual, donde se explican estas disonancias emotivas que 
se despliegan por creencias irracionales que posee una persona sobre su condición de vida; lo cual, ocasiona malestar a nivel cognitivo, conductual y eomocional; asimismo, ambos autores coinciden que la procrastinación se inicia al creer que es imposible desarrollar alguna actividad y presentar poca tolerancia a la frustración para resolverla. Este enfoque cognitivo-conductual es el modelo idóneo para explicar el comportamiento y la procrastinación; además, se encontraron once pasos que parecen ser inevitables para los procrastinadores: 1) desea realizar una tarea, 2) toma la decisión de hacerlo, 3) retrasa innecesariamente, 4) observa la desventaja, 5) continúa posponiendo las tareas, 6) se regaña por procrastinador, 7) continúa postergando, 8) completa tareas en el último minuto, 9) se siente fastidiado, 10) se asegura de no postergar de nuevo; y, 11) poco después, vuelve a procrastinar. Durante este proceso, el individuo experimenta sentimientos desagradables como, ansiedad, depresión y desesperación acompañados de baja autoestima y sentimientos de incapacidad (Ellis y Knaus, 2002).

Esto significa que la persona presenta un razonamiento inadecuado, pues tiene conclusiones erróneas que generalmente no son ciertas. A través de este modelo, se explica como la persona genera estos pensamientos irracionales y la consecuencia de las alteraciones emocionales que repercute sobre la conducta, haciendo que el individuo reaccione de manera contraproducente, principalmente por una baja tolerancia a la frustración, dejando actividades importantes como las tareas de la escuela.

\section{PROPUESTA PREVENTIVA Y ESTRATEGIAS}

Es importante plantear estrategias multidisciplinarias para lograr un oportuno y eficaz desempeño académico del estudiante tanto en la escuela como en la universidad; por ello se requiere una adecuada organización y administración del tiempo. Esto permitirá que los estudiantes se autorregulen, eviten postergar las actividades académicas y dejen de practicar la procrastinación.

Es importante desarrollar talleres para los estudiantes con el objetivo de mantener una actitud positiva, disciplina, concentración y perseverancia, lo cual repercutirá en la mejoría del rendimiento académico (Guerrero, 2017).

También, se puede realizar un plan de acción dirigido a la autorregulación emocional, planificación del tiempo y autoeficacia académica, cuyo efecto repercute en el desarrollo adecuado del aprendizaje (Gómez, Ortiz y Perdomo, 2016).

Además, es necesario establecer un compromiso o acuerdos en relación a la perseverancia y el manejo eficaz del tiempo, con temas enfocados a la resiliencia, la esperanza o el optimismo (González-Brignardelloab y Sánchez-Elvira-Paniagua, 2013). 
Asimismo, se requiere organizar las tareas y dosificarlas en pequeñas metas para gestionar mejor la actividad (Herrera, 2018).

De este modo, se logra promover el desarrollo de habilidades sociales entre los adolescentes escolares, desde los primeros años de la secundaria hasta los últimos tres grados escolares (Torres, 2016).

Igualmente, es substancial identificar los pensamientos relacionados con la procrastinación para disminuir los niveles de angustia y estrés, como las intervenciones cognitivo-conductuales de apoyo enfocadas a dar soporte a esta problemática (Valencia, 2017).

Y por último, es necesario pensar en realizar otras actividades que favorezcan al óptimo desarrollo de los componentes intrapersonal, interpersonal, adaptabilidad y estado de ánimo en los estudiantes (Villanueva, 2019).

Por lo tanto, es importante que los docentes puedan ser guías y que sus funciones estén relacionadas a la motivación e involucramiento con el adolescente para fortalecer la reflexión de superación ante esta dificultad; además, es importante brindar seguridad a los estudiantes para que logren cumplir sus objetivos personales y se sienta competentes en la realización de sus tareas, reflexionando sobre los beneficios de la autorregulación académica y de un aprendizaje sostenido y autónomo. También es necesario que en las horas de tutoría se trate el tema de la procrastinación académica, para que los estudiantes tomen consciencia de las consecuencias negativas en su futuro profesional y laboral; y puedan tener un bagaje de técnicas para un adecuado mecanismo de solución. Finalmente, se sugiere realizar talleres para los padres de familia con el objetivo de orientar sobre la necesidad de establecer adecuadamente sus roles e informar sobre esta postura procrastinadora de sus hijos y así remediarlas positivamente.

\section{CONCLUSIONES}

- El concepto de procrastinación significa posponer actividades en su desarrollo y culminación por preferir otras tareas de interés, y el tiempo es utilizado para socializar, priorizar la recreación o simplemente no hacer tareas.

- Se encontraron dos tipos de procrastinación: procrastinación general, la cual está vinculada a los aspectos de aplazar actividades académicas, laborales y personales; mientras que la procrastinación académica, está enfocada en la postergación de actividades en el plano educativo y universitario.

- Los modelos desarrollados permiten respaldar a la variable procrastinación, ya que enmarca teorías importantes para clarificar su origen y desarrollo. 
- Se evidencian estrategias multidisciplinaria para evitar la práctica de la procrastinación $\mathrm{y}$, que las personas se perjudiquen en su desarrollo personal.

En síntesis, este estudio abre líneas a la investigación científica y profesional vinculados a los factores socio-familiares y los estilos educativos para implementar programas de educación e intervención oportuna en la familia, escuela y del adolescente para realizar modificaciones en el sistema educativo que favorezca a un óptimo aprendizaje y desarrollo de competencias.

\section{REFERENCIAS BIBLIOGRÁFICAS}

Alegre, A. (2014). Autoeficacia y procrastinación académica en estudiantes universitarios de Lima Metropolitana. Propósitos y recomendaciones, 1(2), 57-82. doi.org/ 10.205/ pyc2013vln2.29

Álvarez, O. (2010). Procrastinación general y académica en una muestra de estudiantes de secundaria de Lima metropolitana. Revista persona, 13, 159-177. Recuperado de http://www.redalyc.org/pdf/1471/147118212009.pdf

Angarita, L. (2012). Aproximación a un concepto actualizado de la procrastinación. Revista Ibero Americana de Psicología: Ciencia y Tecnología, 5(2), 85-94. Recuperado de https://revistas.iberoamericana.edu.co/index.php/ripsicologia/article/view/249

Balkis, M. \& Duru, E. (2009). Prevalence of academic procrastination behavior among preservice teachers, and its relationship with demographics and individual preferences. Journal of Theory and Practice in Education, 5(1), 18-32.

Busko, D. (1998). Causes and consequences of perfectionism and procrastination: A Structural equation model. (Tesis de maestría). University of Guelph, Ontario.

Carranza, R. y Ramirez, A (2013). Procrastinación y características demográficas asociados en estudiantes universitarios. Revista de la Universidad Peruana Unión, $3(2), 95-108$.

Chan, L. (2011). Procrastinación académica como predictor en el rendimiento académico en jóvenes de educación superior. Temática psicológica, 7(1), 53-62.

Contreras-Pulache, H., Mori-Quispe, E., Lam-Figueroa, N., Hinostroza-Camposano, W., Rojas-Bolivar, D., Espinoza-Lecca, E., Torrejon-Reyes, E. y Conspira-Cross, C. (2011). Procrastinación en el estudio: exploración del fenómeno en adolescentes escolarizados. Lima Metropolitana, Perú. Revista Peruana de Epidemiología, 15 (3), 1-5. Recuperado de http://www.redalyc.org/pdf/2031/203122771007.pdf

Dominguez, S. y Centeno, S. Procrastinación Académica: validación de una escala en una muestra de estudiantes de una universidad privada. Liberabit. En prensa.

Ellis, A. (1999) Una terapia breve más profunda y duradera. Enfoque teórico de la terapia racional emotivo-conductual. Editorial Paidós.

Ellis, A. \& Knaus, W. (2002). Overcoming procrastination. New York: New 
Ferrari, J., Johnson, J. \& McCown, W. (1995). Procrastination and task avoidance. New York: Plenum Press.

Ferrari, J., y Díaz-Morales, J. (2007). Perceptions of self concept and self presentation by procrastinators: Further evidence. The Spanish Journal of Psychology, 10, 91-96. Recuperado de https://www.sciencedirect.com/science/article/pii/ S0092656699922614

García-Ayala, C. (2016). Comprendiendo la procrastinación con el Modelo ABC de Albert Ellis. ResearchGate, 2(1), 7-5. Recuperado de https://www.researchgate.net/ publication/264421119

Gómez, C., Ortiz, N. y Perdomo, L. (2016). Procrastinación y factores relacionados para su análisis en la educación superior. Revista de investigaciones, 7(1), 32-39.

González-Brignardello, M. y Sánchez-Elvira-Paniagua, A. (2013). ¿Puede amortiguar el engagement los efectos nocivos de la procrastinación académica? Acción Psicológica, 10(1), 115-134. http://dx.doi.org/10.5944/ap.10.1.7039

Guerrero, S. (2017). Clima social escolar y procrastinación en estudiantes del primer y segundo año de secundaria de una Institución Educativa de acción conjunta del distrito de Florencia de Mora - Trujillo (Tesis de Maestría). Universidad Privada Antenor Orrego, Trujillo.

Herrera, T. (2018). Por qué tu cerebro te hace procrastinar. The new work times ES. Recuperado de https:/www.nytimes.com/es/2018/07/11/por-que-tu-cerebro-te-haceprocrastinar/

Natividad, S. (2014). Análisis de procrastinación en estudiantes universitarios. (Tesis Doctoral). Universidad de Valencia, Valencia.

Quant, D. y Sánchez, A. (2012). Procrastinación académica: concepto e implicaciones. Revista Vanguardia Psicológica Clínica Teórica y Práctica 3(1), 45-59.

Rosário, P., Costa, M., Nuñez, J., Gonzales-Pienda, J., Solano, P. y Valle, A. (2009). Academic Procrastination: Associations with personal, school, and family variables. The spanish Journal of Psychology, 12, 118-127. Recuperado de https://search. proquest.com/docview/274589208?accountid $=12268$

Rothblum, E., Solomon, J. \& Murakami, J. (1986). Affective, cognitive, and Behavioral differences between high and low procrastinators. Journal of counseling psychology, 33, 387-394.

Sirois, F. \& Pychyl, T. (2013). This is a repository of Procrastinacition and the Priority of short-tem Mood regulation: Consequences for future Self. Social and personality Psychology Compass, 7(2), 115-127. https://doi.org/10.1111/spc3.12011

Skinner, E. \& Belmont, M. (1993). Motivation in the classroom: Reciprocal effects of teacher behavior and student engagement across the school year. Journal of Educational Psychology, 85, 571-581. http://dx.doi.org/10.1037//0022-0663.85.4.571

Steel, P. (2007). The nature of procrastination: A meta-analytic and theoretical review of quintessential self-regulatory failure. Psychological Bulletin, 133(1), 65-94. 
Torres, A. (2016). Relación entre habilidades sociales y procrastinación en adolescentes escolares. (Tesis de Maestría). Universidad Cooperativa de Colombia, Santa Marta.

Valencia, Y. (2017). Funciones ejecutivas, procrastinación académica y rendimiento académico en estudiantes de secundaria. (Tesis de Maestría). Pontificia Universidad Católica del Perú, Lima.

Valle, M. (2017). Procrastinación académica en estudiantes de Secundaria de la Institución Educativa N¹26 “Javier Pérez de Cuéllar”, Lima. (Tesis de Licenciatura). Universidad Inca Garcilaso De La Vega, Lima.

Villanueva, F. (2019). Inteligencia emocional y procrastinación académica en estudiantes de cuarto de secundaria de una institución educativa. (Tesis de Maestría). Universidad César Vallejo, Lima.

Wolters, C. (2003). Entendimiento de la procrastinación del self-regulación de perspectiva. Revista de educación Psicológica, 95(1), 179-187. 
